\title{
PARASITISMO OCULAR EN PATAGONOTOTHEN CORNUCOLA (PISCES: NOTOTHENIIDAE) POR UN COPÉPODO SIPHONOSTOMATOIDA (PENNELLIDAE) EN LA REGIÓN DE MAGALLANES, CHILE
}

\author{
OCULAR PARASITISM IN PATAGONOTOTHEN CORNUCOLA (PISCES: NOTOTHENIIDAE) BY \\ A SIPHONOSTOMATOID COPEPOD (PENNELLIDAE) AT THE MAGALLANES REGION, CHILE
}

Juan I. Cañete $e^{1}$, Mathias Hüne ${ }^{2}$, Daniela Haro ${ }^{1}$, Alvaro Medina ${ }^{1}$, Pamela González ${ }^{1} \&$ Iván A. Cañete ${ }^{3}$

\begin{abstract}
Penellidae copepods possess a body of saccular shape and are found almost exclusively in the eyes of some demersal fishes (e.g. flat-fish). This report deals with the presence of a parasitic, siphonostomatoid copepod in the eyes of Patagonotothen cornucola at Porvenir Bay, Strait of Magellan, Chile shallow areas $(<$ $1 \mathrm{~m}$ depth) (5318'30' S) (70²3'00' W), giving some details on the level of prevalence ( $\mathrm{N}=131$ individuals, $<60 \mathrm{~mm}$ TL; $11.5 \%$ parasitized). Additionally, is detected this ecological relationship in other two Magellan locations.
\end{abstract}

Key words: Parasitism, Nototheniidae, Phrixocephalus, Copepod, Subantarctic megafauna.

\section{RESUMEN}

Los copépodos parásitos sifonostomatoides pertenecientes a la familia Penellidae poseen un cuerpo de aspecto sacular y se encuentran casi exclusivamente en los ojos de algunas especies de peces demersales (e.g. peces planos). En el presente trabajo se registra la presencia de un parásito de estas características, no identificado aún a nivel específico, que habita en uno o ambos ojos del pez nototheniido Patagonotothen cornucola capturados dentro de una matriz de algas filamentosas en zonas someras ( $<1 \mathrm{~m}$ profundidad) de Bahía Porvenir, Estrecho de Magallanes, Chile (agosto, 2010 y diciembre, 2011) (53²18'30' S, 70²3'00 O) El 11,5\% de 131 individuos examinados estaba parasitado Todos los ejemplares presentaron un tamaño < $60 \mathrm{~mm}$ LT;). Se adicionan otros dos sectores de la región de Magallanes donde también se observó dicha relación ecológica.

Palabras clave: Parasitismo, Nototheniidae, Phrixocephalus, Copepoda, Megafauna subantártica.

\footnotetext{
${ }^{1}$ Facultad de Ciencias, Universidad de Magallanes, Punta Arenas, Chile.

${ }^{2}$ Instituto de Ecología y Biodiversidad, Facultad de Ciencias, Universidad de Chile, Casilla 653, Santiago, Chile.

${ }^{3}$ Facultad de Ciencias, Universidad de Magallanes, Punta Arenas, Chile. ivan.canete@umag.cl
} 


\section{INTRODUCCIÓN}

Durante una campaña de análisis de la megafauna bentónica de bahía Porvenir, Tierra del Fuego, Chile (Fig. 1), se capturaron algunos peces identificados como Patagonotothen cornucola (Richardson, 1844), los cuales se encontraban con un parásito fijado en uno de sus ojos. $P$. cornucola es una especie endémica de las aguas costeras subantárticas de Sudamérica, habitando desde el seno de Reloncaví (4139' S), por el Pacífico sur, hasta las costas patagónicas del Atlántico e islas Malvinas/Falkland (Lloris \& Rucabado, 1991; Reyes \& Hüne, 2012). Esta especie es abundante en el intermareal rocoso y submareal somero (hasta $15 \mathrm{~m}$ de profundidad) de la región de Magallanes, habitando principalmente en bosques de Macrocystis pyrifera (Linnaeus) Agardh, 1820 (Moreno \& Jara, 1984; Lloris \& Rucabado, 1991; Hüne \& Ojeda, 2012).

El objetivo del presente trabajo es dar a conocer la presencia de un copépodo parásito, tentativamente identificado a nivel genérico como Phrixocephalus, parásito ocular del pez subantártico perteneciente al género Patagonotothen recolectado en algunas localidades someras de la región de Magallanes, Chile.

\section{MATERIAL Y METODOS}

Área de estudio

El área de estudio incluye 4 localidades visitadas entre los años 2008 y 2011 en la zona
P. cornucola fue la única especie de este género presente en esta zona, pese a que se reconoce la presencia de 13 especies en el estrecho de Magallanes (Sielfeld \& Vargas, 1999; Reyes \& Hüne, 2012). En esta bahía, los peces viven en una matriz de algas filamentosas constituidas por al menos 4 especies de algas (A. Mansilla, Com. Pers.), que conforman uno de los paisajes submareales más típicos de la cabeza de esta bahía.

Las capturas de los peces se realizaron mediante el uso de una red de arrastre tipo Agassiz de $100 \mathrm{~cm}$ de ancho de boca, $30 \mathrm{~cm}$ de alto y provisto con una red y copo de $4 \mathrm{~mm}$ de trama, siendo arrastrada aproximadamente entre 500 y 700 $m$ de distancia a una velocidad de la embarcación de 1,5 nudos. Este muestreo formó parte de una investigación sobre la dinámica espacial y estacional de la megafauna presente en bahía Porvenir, Chile (Cañete et al. 2010; González-Wevar et al. 2012).

Otro grupo de peces se observaron en localidades ubicadas en el sector occidental del Estrecho de Magallanes (Tabla 1; Fig. 1), detectando casos de parasitismo en $P$. cornucola mediante buceo autónomo, efectuando observaciones submarinas in situ y fotografiando los peces utilizando una cámara fotográfica Nikon D200 y un lente Micro Nikkor de $60 \mathrm{~mm}$. Los muestreos mediante buceo se realizaron en un sector rocoso de 3 a $20 \mathrm{~m}$ de profundidad aledaño a bosques de $M$. pyrifera.

Data oceanográfica

En bahía Porvenir se hizo registro de la temperatura en la columna de agua mediante una

Tabla 1. Registros de parasitismo ocular en el pez subantártico Patagonotothen cornucola en distintas zonas de la región de Magallanes. Entre paréntesis se indica el número de parásitos por pez.

\begin{tabular}{lllll}
\hline Zona colecta & Fecha Colecta & Profundidad $(\mathrm{m})$ & Coordenadas $^{\prime}$ & $\mathrm{N}^{\circ}$ ejemplares \\
\hline Estero Staples & Enero, 2008 & 10 & $54^{\circ} 02^{\prime} \mathrm{S} ; 71^{\circ} 14^{\prime} \mathrm{O}$ & $1(2)$ \\
Estero Staples & Agosto, 2010 & 5 & $54^{\circ} 02^{\prime} \mathrm{S} ; 71^{\circ} 14^{\prime} \mathrm{O}$ & $1(2)$ \\
Puerto Hambre & Agosto, 2010 & 10 & $53^{\circ} 39^{\prime} \mathrm{S} ; 72^{\circ} 13^{\prime} \mathrm{O}$ & $1(2)$ \\
Bahía Porvenir & Agosto, 2010 & 1 & $53^{\circ} 18^{\prime} \mathrm{S} ; 70^{\circ} 23^{\prime} \mathrm{O}$ & $15(1)$ \\
Estero Lyell & Diciembre, 2011 & 12 & $54^{\circ} 01^{\prime} \mathrm{S} ; 71^{\circ} 22^{\prime} \mathrm{O}$ & $1(2)$ \\
\hline
\end{tabular}

costera del estrecho de Magallanes, Chile (Fig. 1). Un grupo de peces se capturaron en una zona so-

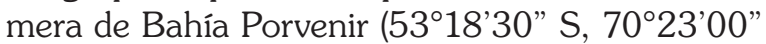
O; $1 \mathrm{~m}$ profundidad), Tierra del Fuego, Chile. El pez sonda oceanográfica Sea Bird Mod. 19, o con un termómetro digital que permitió registrar la temperatura superficial, considerando que la columna de agua ha sido descrita como homogénea desde el 


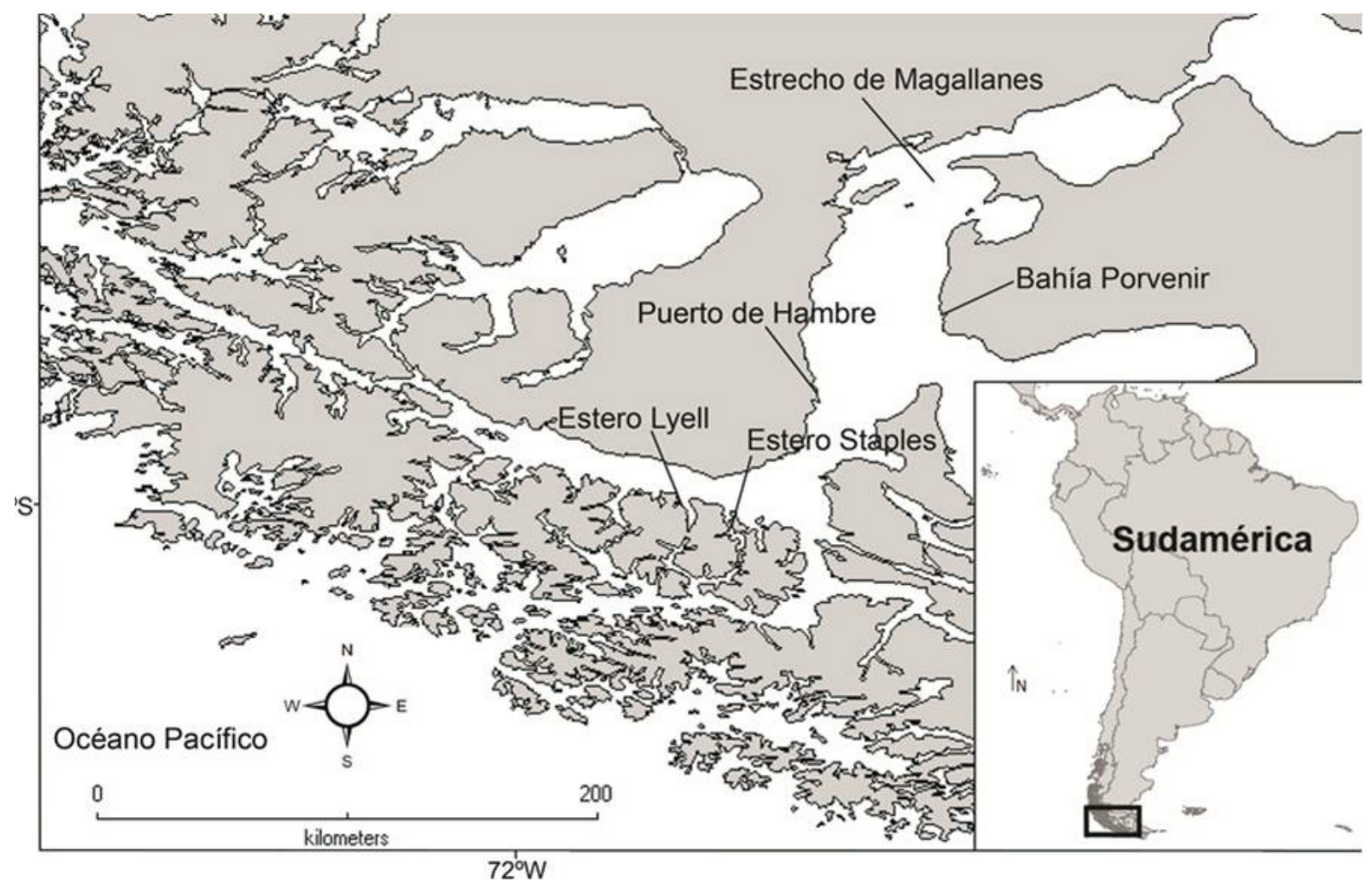

Fig. 1. Localidades donde se ha registrado parasitismo ocular por copépodos representantes familia Pennellidae en el pez Patagonotothen cornucola en la región de Magallanes, Chile.

punto de vista térmico, halino y de oxigenación, tal como otras zonas costeras someras de esta región (Valdenegro \& Silva, 2003; Cañete et al., 2008). En las otras tres localidades la temperatura se registró mediante un sensor incorporado en el computador de buceo Suunto D4i.

Descripción del parásito ocular

Algunas características taxonómicas usadas para describir las diferentes especies de copépodos parásitos oculares se basan en la morfología del disco de fijación: éste consiste de numerosas raicillas entrelazadas originadas a partir del cefalotórax del parásito y fijado en el coroide del ojo del huésped. Alrededor del disco, se encuentra un gran hematoma o laguna donde se almacena sangre. Perkins (1994), mediante el uso de la microscopía electrónica, reveló que las raicillas están constituidas por una cutícula trilaminar: epicutícula externa, una epicutícula interna y una procutícula externa. La superficie externa de la epicutícula está cubierta de microvellosidades que toman contacto con los tejidos del huésped. En la procutícula es posible observar depósitos de cristales fijados a ella. Internamente, las raicillas se componen de al menos tres tipos de células grandes: i) células epiteliales en la capa interna de la procutícula que contiene numerosas mitocondrias; ii) células de forma de estrella cuyo citoplasma estuvo dominado por el retículo endoplásmico rugoso o grandes vacuolas; iii) las células ovoides que contienen gránulos proteicos de composición química desconocida. Las observaciones de Perkins revelaron que las raicillas son estructuralmente complejas y sugieren que el disco de fijación puede participar activamente en el intercambio molecular entre parásito y huésped (Perkins, 1994).

Los peces fueron trasladados al Laboratorio de Oceanografía Biológica, campus Instituto de la Patagonia, Universidad de Magallanes, donde fueron identificados, medidos y pesados. La identificación se realizó mediante las siguientes referencias bibliográ- 
ficas especializada en grupos taxonómicos de peces del sur de Chile: Navarro \& Pequeño (1979), Lloris \& Rucabado (1991), Pequeño (1989, 1999, 2000), Reyes \& Hüne (2012). Los peces que presentaron parasitismo ocular fueron separados y analizados mediante un microscopio estereoscópico, con el fin de identificar taxonómicamente al parásito, utilizando la siguiente literatura: Kabata (1967; 1969 \& 1981) para copépodos Penellidae representantes del género Phrixocephalus. Se midió la longitud total de cada pez y se contabilizó el número de parásitos y su presencia en uno o ambos ojos. Fotografías del copépodo parásito fueron remitidas al Dr. Juan Carvajal (Universidad Los Lagos, Osorno, Chile) para una identificación preliminar, quién además sugirió la posibilidad de pertenecer al género Phrixocephalus y aportó literatura al respecto.

\section{Depósito de ejemplares}

Dos especímenes recolectados en bahía Porvenir en agosto 2010 se confundieron con hirudíneos y se enviaron para la identificación experta del Dr. Eugene Burreson (Virginia Institute of Marine Science, Williams \& Mary College, Estados Unidos); en el año 2011 se comunicó que correspondían a un copépodo parásito y dichos ejemplares quedaron depositados en dicha institución. El material restante
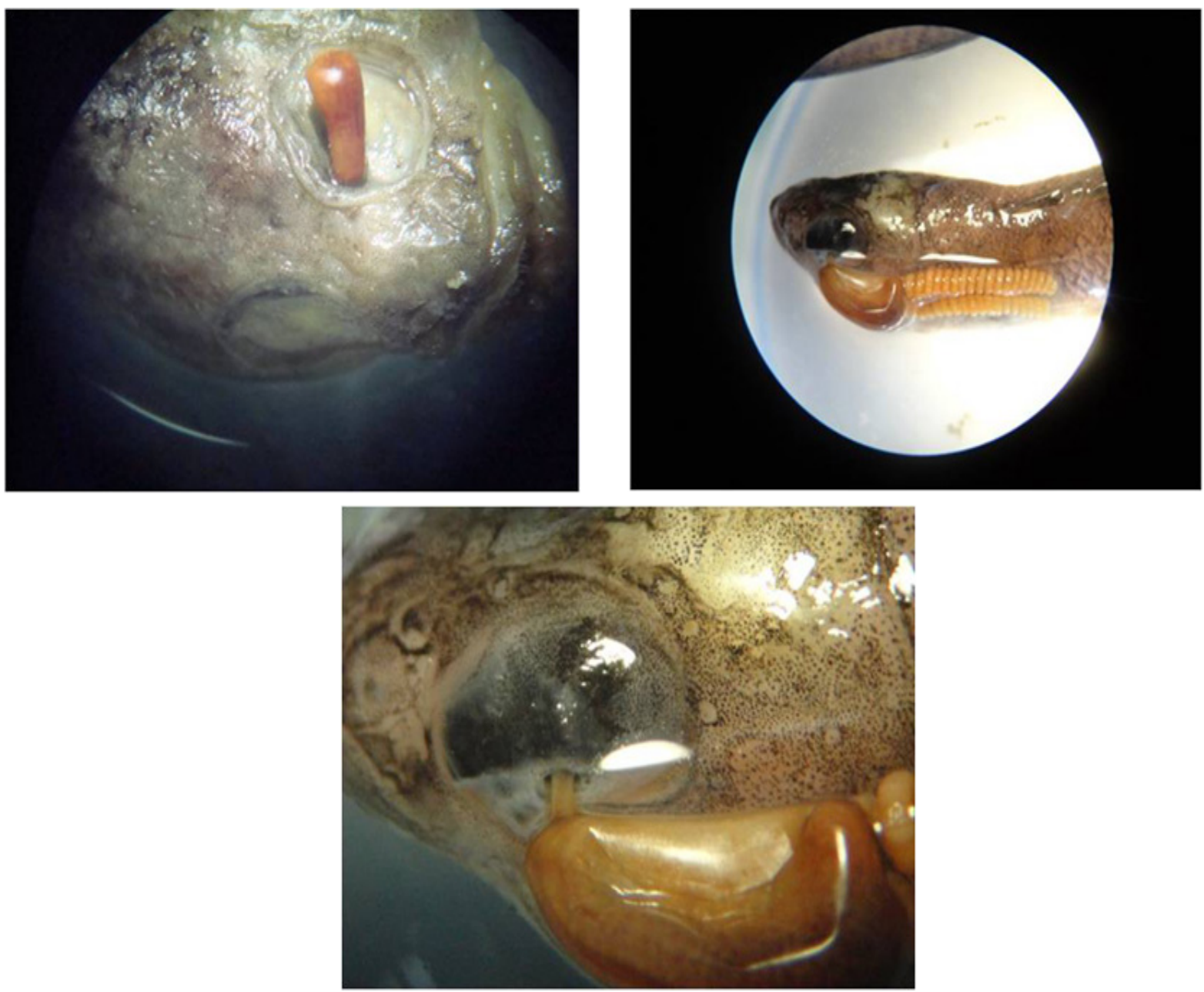

Fig. 2.- Parasitismo de Phrixocephalus sp en el ojo del pez subantártico Patagonotothen cornucola en bahía Porvenir, Tierra del Fuego, Chile, en dos períodos estacionales; a) espécimen inmaduro (agosto, 2010), b) espécimen reproductivamente maduro (diciembre 2011); c) detalle del pedúnculo del disco de fijación en el ojo izquierdo (diciembre, 2011). 

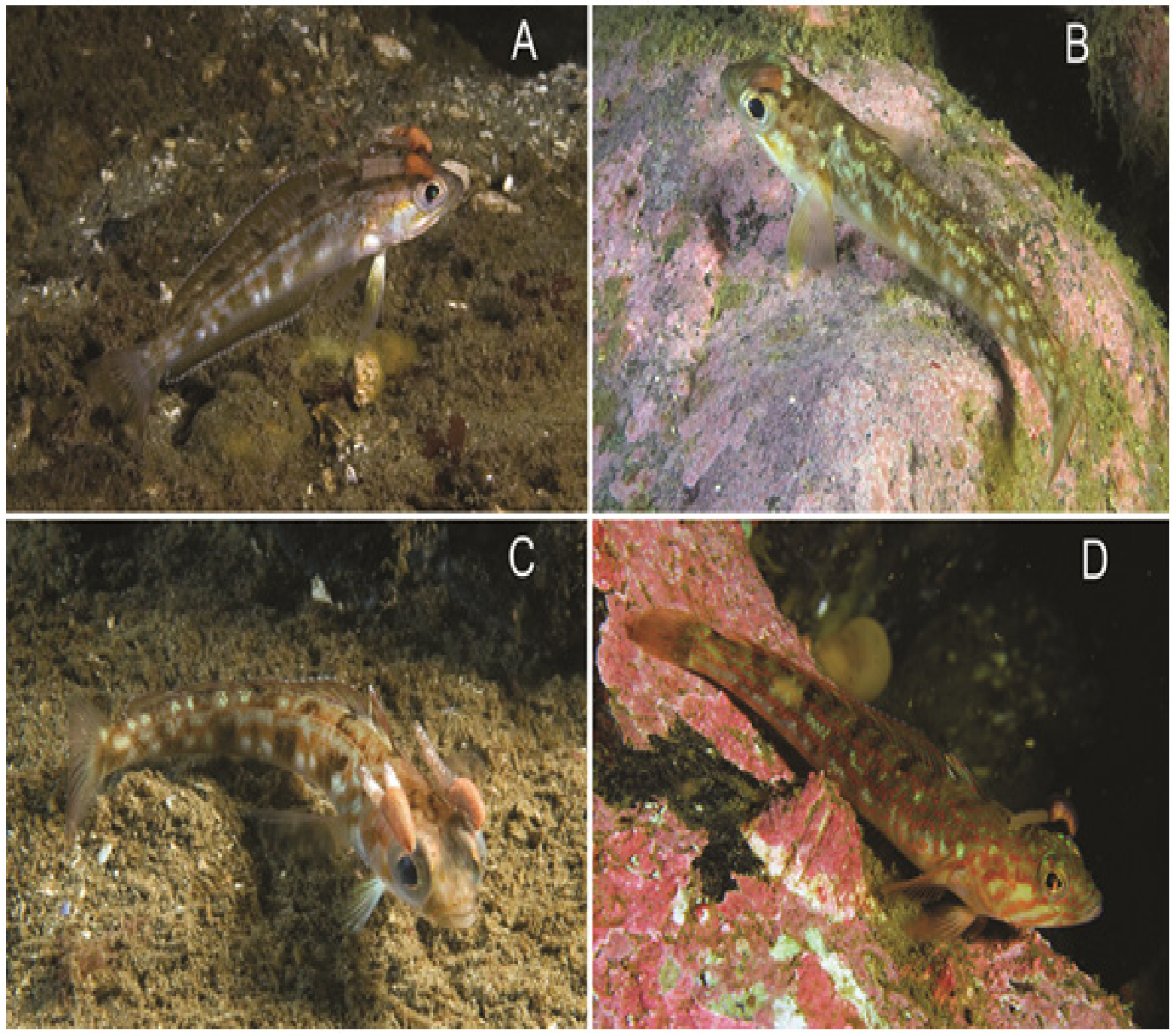

Fig 3.- Parasitismo de Phrixocephalus sp en ambos ojos del pez subantártico Patagonotothen cornucola en tres sectores del estrecho de Magallanes, Chile. A y B= Estero Staples, $\mathrm{C}=$ Puerto de Hambre, $\mathrm{D}=$ Estero Lyell. Estos peces miden entre 6 y $8 \mathrm{~cm}$ (Fotografías de Mathias Hüne).

de las diferentes localidades, peces y sus respectivos parásitos, se depositaron en la Sala de Colecciones del Instituto de la Patagonia bajo el número de registro 900142-CIIP-CHO-900137.

\section{RESULTADOS}

En bahía Porvenir se efectuaron 5 campañas de observaciones (agosto, 2010, enero, mayo, diciembre 2011 y marzo 2012), sin embargo en el presente estudio se informa de los resultados obte- nidos sólo en dos de estas campañas: agosto 2010 y diciembre 2011. En agosto 2010 se recolectaron 27 peces, 5 de los cuales presentaban Phrixocephalus en estado de inmadurez sexual (Fig. 2), en uno o ambos ojos, pero siempre un parásito por ojo. En bahía Porvenir, durante la campaña de agosto 2010 se registró una temperatura superficial de la columna de agua que varió entre 1,2 y $2,1^{\circ} \mathrm{C}$, mientras que en diciembre 2011 se recolectaron 36 peces, 7 de los cuales se encontraban con uno de sus ojos parasitados; en esta campaña, la temperatura superficial 
fue de $14^{\circ} \mathrm{C}$

En el sector occidental del estrecho de Magallanes, se contabilizaron 4 casos de parasitismo ocular de Phrixocephalus en el pez nototénido P. cornucola (Tabla 1). Los ejemplares recolectados en agosto 2010 y en diciembre 2011 en bahía Porvenir corresponden a individuos juveniles $(<60 \mathrm{~mm}$ longitud total). Los especímenes fotografiados en el Estero Staples, Estero Lyell y Puerto de Hambre corresponden a individuos adultos (80 a $100 \mathrm{~mm}$ ) (Fig. 3).

Por otra parte, los registros mediante fotografía submarina permitieron identificar la presencia del parasito en ambos ojos (Estero Staples, Enero 2008 y Puerto de Hambre), o solamente en un ojo (Estero Staples, Agosto 2010 y Estero Lyell) (Fig. 3). Además, la temperatura del agua a la profundidad de registro presentó su mayor valor en enero $2008\left(10,3^{\circ} \mathrm{C}\right)$, mientras que la menor se obtuvo en agosto $2010\left(6^{\circ} \mathrm{C}\right)$, ambos registros efectuados en el Estero Staples.

\section{DISCUSIÓN}

El ojo de los vertebrados es inusual como hábitat para otros invertebrados. Algunas species de copépodos han aprovechado (Kabata, 1970; Munz, 1971; Wilcock \& Dukes, 1989; Easter, 1992; Cameron \& Easter, 1995; Bellhorn, 1997; Perkins \& Gartman, 1997; Jones, 1998; Bone et al., 1999; Sandtröm, 1999; Evans, 2004; Cherel \& Boxshall, 2004; Blaylock et al., 2005; Dahm et al., 2007; Otsuka et al., 2007; Corchuelo et al., 2012) este hábitat de maneras diferentes: Ommatokoita elongate infecta sólo superficialmente los ojos de dos especies de tiburones y "ramonea" las células epiteliales de la córnea y la conjuntiva, lo que limita su exposición a la inmunorespuesta del huésped, afectando sólo parcialmente la visión de éste debido a una limitada respuesta inmune de la córnea. Benz et al. (2002) considera el ojo del tiburón un ambiente menos hostil que otras partes del cuerpo, siendo ventajoso para O. elongata. De hecho, varias generaciones de $O$. elongata pueden utilizar el mismo ojo (Borucinska et al., 1998; Benz et al., 2002).

En aquellos casos donde participan miembros del género Phrixocephalus ( $P$. cincinnatus), la cornea se encuentra escasamente alterada, pero en casos graves de infestación se ha observado vaso- dilatación, edemas y una mayor permeabilidad que permite el acceso de la sangre y otros fluidos hacia el parásito. Además, Kabata (1969) propuso que P. cincinnatus (Wilcock \& Dukes, 1989) expulsa las enzimas digestivas a través del ano para digerir el ojo y así facilitar la extrusión de las cadenas de huevos. Esto podría contribuir a la destrucción interna de los ojos afectados. Kabata (1967) indica que la ceguera y la total destrucción del ojo es el resultado inevitable de la infección por $P$. cincinnatus. La muerte del parásito, ya sea por falta de desarrollo o senescencia, resulta en la destrucción total del ojo (Kabata \& Forrester, 1974). En nuestro caso, se observa una grave destrucción de las capas superficiales generada por la fijación del disco de adhesión (Fig. 2c).

Es importante destacar que en Chile existe sólo una referencia sobre la presencia del género Phrixocephalus. Atria (1977) se refiere a la presencia de un miembro del género en el pez costero Pinguipes chilensis (rollizo), pero no señala el órgano parasitado ni el estado de desarrollo (Muñoz \& Olmos, 2007). Sin embargo, se han detectado representantes de otros géneros pertenecientes al orden Siphonostomatoidea en aguas chilenas y magallánicas. Entre estos se citan Caligus y Sphyrion. El primero es considerado una plaga en centros de cultivo de salmónidos (Carvajal et al., 1998) y el segundo ha sido detectado en peces demersales tales como el congrio dorado y merluza austral (Genypterus blacodes y Merluccius australis) en la zona austral de Chile como también en la costa de las Islas Malvinas o Falkland (Brickle et al., 2005). Algunos caracteres diagnósticos para identificar los representantes de este orden son: i) segmentación corporal reducida o vestigial, ii) anténulas modificadas, vestigiales o elongadas, pudiendo finalizar en un tipo de pinza utilizado para adherirse al cuerpo del huésped; iii) mandíbulas encerradas en un tipo de sifón o tubo estructurado mediante la fusión del labrum y labium; iv) maxilas pueden estar modificadas para fijarse al huésped; apéndices toráxicos pueden presentar aspecto normal y ser utilizados para la natación o pueden estar ampliamente modificados (Bush et al., 2001; Rodhe et al., 1998)

Considerando las condiciones oceanográficas y las profundidades de recolección de $P$. cornucola en los cuatro lugares visitados en este estudio, se puede señalar que estos valores son típicos de estas 
zonas del estrecho de Magallanes (Panella et al., 1991; Valdenegro \& Silva, 2003). Según Silva et al. (1995, 1998) y Sievers et al. (2002), las aguas del estrecho de Magallanes con una salinidad $<32$ psu se pueden clasificar como una condición típica de estuario.

Otro factor común entre las zonas de recolección de Phrixocephalus es la presencia de praderas de la macroalga parda Macrocystis pyrifera. Es importante destacar que estas praderas proporcionan un hábitat para una variedad de organismos marinos, contribuyendo además a disminuir la fuerza del oleaje y afectando las condiciones hidrodinámicas locales (Ekman et al., 1989, Balch \& Scheibling, 2000). Estos efectos, podrían aumentar la retención de formas larvales de copépodos parásitos como de isópodos (Cañete et al., 2008), tal como se ha postulado para otros estadios larvarios de invertebrados bentónicos así como del detritus y la materia orgánica (Eckman et al. 1989; Duggins et al. 1990, Pakomov et al. 2002). Estos antecedentes fueron propuestos por Cañete et al., (2008) para explicar los altos niveles de infestación del isópodo endoparásito Pseudione tuberculata en juveniles del crustáceo Lithodes santolla en una localidad de la región de Magallanes.

En bahía Porvenir, el hábitat que podría facilitar la agregación de larvas de este parásito estaría representado por una pradera de algas filamentosas (hasta $45 \mathrm{~kg}$ por $700 \mathrm{~m}$ de área barrida), sumado a una columna de agua de escasa profundidad $(<$ $2 \mathrm{~m}$ ). Otro factor que podría ayudar a incrementar la colonización de los parásitos sobre los individuos juveniles de P. cornucola en Bahía Porvenir sería el comportamiento gregario de los peces, su estilo de vida bentodemersal debido a la ausencia de una vejiga natatoria y a la gran abundancia que alcanzan dentro de la matriz de algas filamentosas. En agosto 2010 se registró una abundancia total de 32 peces y de 42 en diciembre 2011.

Por otro lado, cabe destacar la presencia de dos ejemplares parásitos en cada uno de los ojos de P. cornucola en el Estero Staples (Fig. 3 A). Este registro se contrapone a lo observado en Bahía Porvenir donde sólo se observó un Phrixocephalus por ojo. Además, considerando el rango de tallas que presenta $P$. cornucola que varía entre $1,5-11,0 \mathrm{~cm}$ de longitud total, con una talla máxima de $13 \mathrm{~cm}$ (Lloris \& Rucabado, 1991; Reyes \& Hüne, 2012), se puede señalar que los ejemplares parasitados en Bahía Porvenir correspondieron a individuos juveniles y sub-adultos. En este sentido, los peces de bahía Porvenir presentaban tallas inferiores a 6 $\mathrm{cm}$ de longitud total, mientras que los ejemplares de las otras localidades no superaban los $8 \mathrm{~cm}$ de longitud total. Estos resultados son congruentes con las observaciones realizadas en ejemplares adultos ( $>$ a $10 \mathrm{~cm}$ longitud total), donde no se ha registrado la presencia de parásitos oculares (M. Hüne, obs pers.), lo que podría hacer suponer un mayor parasitismo sobre los ejemplares juveniles y sub-adultos. Sin embargo, se requieren estudios adicionales que permitan incluir diferentes rangos de tallas para una mejor compresión de la existencia de una invasión ontogenética a la zona ocular de los peces nototénidos del género Patagonotothen.

Se propone que dadas las condiciones de facilidad de recolección de muestras del pez $P$. cornucola en estado juvenil en bahía Porvenir, mediante redes o con rastras, se considere este lugar como un área experimental para conocer mayores antecedentes del ciclo de vida del parásito y del huésped tales como cuándo se produce la fijación al huésped, las condiciones ambientales que lo hacen madurar reproductivamente y aumentar la población, como logran dispersarse en estos cuerpos de agua subantárticos o si existen más especies de peces afectadas o es específica de miembros del género Patagonotothen. Sin embargo, los niveles de infestación reportados en el presente estudio se pueden considerar de escasa magnitud en comparación a los niveles de infestación detectados por otras especies de copépodos parásitos sifonomastoideos que afectan a salmónidos y cóngridos en la zona austral de Chile, donde se han detectado niveles de infestación > 40\% (Carvajal et al., 1998; Brickle et al., 2003).

Eubrachiella antarctica ha sido citada como una de las escasas especies de copépodos que parasitan a otros miembros de la familia Nototheniidae, en particular a Dissostichus elegenoides. Brickle et al. (2005), en muestras de peces capturadas en distintas islas subantárticas (Georgia del Sur, Bouvet, Prince Edward, Crozet, Kerguelen, Macquarie y Heard) señalan la presencia de copépodos, pero no hacen referencia al género Prixocephalus, pese a haber detectado otras 31 especies de parásitos.

Finalmente, dada la proximidad de los centros de cultivo de salmónidos en la zona austral 
de Chile a la zona costera de algunas de las áreas acá investigadas, es importante destacar que se hace necesario vigilar la presencia de este parásito por cuanto podría afectar a distintos estadios de desarrollo de los salmones. La empresa Marine Harvest Canadá lo tiene incorporado en la lista de enfermedades parasitarias que afectan a los cultivos de peces planos y se discute si se puede relacionar con la presencia de centros de cultivo de salmónidos ya que su hallazgo en la Columbia Británica es reciente (2010) (http://marineharvestcanada.com).

\section{AGRADECIMIENTOS}

Este trabajo se desarrolló mediante un programa interno financiado por la Dirección de Investigación y Postgrado de la Universidad de Magallanes (PR-F2-01CNR-10) y se culminó gracias al Proyecto CIMAR 16-Fiordos (C16F 10-014) financiado por el Comité Oceanográfico Nacional de Chile (CONA) y el Programa Interno (PR-F2-01CNR-12). Se agradece a ambas instituciones. También se agradece al Prof. Juan de Dios Carvajal (Universidad de Los Lagos, Osorno, Chile), quien sugirió inicialmente que el copépodo podría pertenecer al género Phrixocephalus y aportar literatura al respecto.

\section{LITERATURA CITADA}

Almanza, V., A. H. Buschmann M. C. HernándezGonzález \& L. A. Henríquez (2012). Can giant kelp (Macrocystis pyrifera) forests enhance invertebrate recruitment in southern Chile? Marine Biology Research 8, 855-864.

Atria, G. (1977). Lista de copépodos asociados a organismos marinos en Chile (Caligoidea, Lerneapoidea, Cyclopoida). Noticiario Mensual del Museo Nacional de Historia Natural 21: 2-7.

Balch, T. \& R. E. Scheibling (2000). Temporal and spatial variability in settlement and recruitment of echinoderms in kelp beds and barrens in Nova Scotia. Marine Ecology Progress Series, 205, 139-154

Bellhorn, R. (1997). Retinal nutritive systems in vertebrates. Seminars in Avian and Exotic Pet Medicine 6, 108-118.

Benz, G. W., J. D. Borucinska, L. F. Lowry \& H. E. Whiteley (2002). Ocular lesions associated with attachment of the copepod Ommatokoita elongate (Lernaeopodidae: Siphonostomatoida) to corneas of Pacific sleeper sharks Somniosus pacificus captured off Alaska in Prince William Sound. Journal of Parasitology 88, 474-481. Blaylock, R. B., R. M. Overstreet \& A. Morton (2005). The pathogenic copepod Phrixocephalus cincinnatus (Copepoda: Pennellidae) in the eye of arrow tooth flounder, Atherestes stomias, and rex sole, Glyptocephalus zachirus, from British Columbia. Bulletin of European Association of Fish Pathology 25, 116-123.

Bone, Q., N. B., Marshall \& J. H. S. Blaxter (eds.) (1999). Biology of Fishes. Tertiary Level Biology, Stanley Thornes Publishers Ltd., Cheltenham.

Borucinska, J. D., G. W., Benz \& H. E. Whiteley. (1998). Occular lesions associated with attachment of the parasitic copepod Ommatokoita elongata (Grant) to corneas of Greenland sharks, Somniosus microcephalus (Bloch \& Schneider). Journal of Fish Diseases 21, 415-422.

Brickle, P., N. G. Buxton \& E. Villalón (2003). Infection of Sphyrion laevigatum (Copepoda: Sphyriidae) on Genypterus blacodes (Pisces: Ophidiidae) from the Falkland Islands, South Atlantic. Journal of Parasitology 89, 242-244.

Brickle P., K. MacKenzie \& A. Pike (2005). Parasites of the Patagonian toothfish, Dissostichus eleginoides Smitt 1898, in different parts of the Subantarctic. Polar Biology 28, 663-671.

Bush, A. O., Fernández J. C., G. W., Esch \& J. R., Seed (2001). Factors influencing parasite population. In: Parasitism. The diversity and ecology of animal parasites. Cambridge University Press, Cambridge, 345 p.

Cameron, D. A. \& S. S. Easter (1995). Cone photoreceptor regeneration in adult fish retina: Phenotypic determination and mosaic pattern formation. The Journal of Neuroscience 15, 2255-2271.

Cañete, J. I., C. A. Cárdenas, S. Oyarzún, J. Plana, M. Palacios \& M. Santana (2008). Pseudione tuberculata Richardson, 1904 (Isopoda: Bopyridae): a parasite of juveniles of the king crab Lithodes santolla (Molina, 1782) (Anomura: Lithodidae) in the Magellan Strait, Chile. Revista de Biología Marina y 
Oceanografía 43, 265-274.

Carvajal, J., L. González \& M. George-Nascimento (1998). Native sea lice (Copepoda: Caligidae) infestation of salmonids reared in net pen systems in southern Chile. Aquaculture 166, 241-246.

Cherel, Y. \& G. A. Boxshall (2004). Sarcotretes (Copepoda: Pennellidae) parasitizing myctophid fishes in the Southern Ocean: new information from seabird diet. Journal of Parasitology 90, 1288-1292.

Corchuelo, S., M. L. Caldas, R. Peña, H. Hurtado \& M. O. Tovar (2012). Descripción histológica del ojo de Panaque nigrolineatus (Siluriformes: Loricariidae) y sus implicaciones ecomorfológicas. Revista Chilena de Historia Natural 85, 199-208.

Dahm, R., H. B. Schonthaler, A. S. Soehn, J. Marle \& G. F. J. Vrensen (2007). Development and adult morphology of the eye lens in the zebrafish. Experimental Eye Research 85, 74-89.

Devadas, M., K. Sugawara, Y. Shimada, K. Sugitane, Z. Wu Liu et al. (2000). Slow recovery of retinal ganglion cell's soma size during regeneration. Neuroscience Research 37, 289-297.

Duggins, D. O., J. E. Eckman \& A. T. Sewell (1990). Ecology of understory kelp enviroments. 2. Effects of kelp on recruitment of benthic invertebrates. Journal of Experimental Marine Biology and Ecology 143, 27-45.

Easter, S. S. (1992). Retina growth in foveated teleosts: Nasotemporal asymmetry keeps the fovea in temporal retina. The Journal of Neuroscience 12, 2381-2392.

Eckman, J. E., D. O. Duggins \& A. T. Sewell (1989). Ecology of understory kelp environments. 1. Effects of kelp on flow and particle transport near the bottom. Journal of Experimental Marine Biology and Ecology 129, 173-187

Evans, B. (2004). A fish's eyes view of habitat change. En: G. Von Der Emde, J. Mordans \& B. G. Kapoor (eds.), the senses of fish, adaptations for the receptions of natural stimuli. Kluwer Academic publishers, London.

González-Wevar, C. A., A. Díaz, K. Gerhard, J. I. Cañete \& E. Poulin (2012). Divergence time estimations and contrasting patterns of genetic diversity between Antarctic and southern South America benthic invertebrates. Revista
Chilena de Historia Natural 85, 445-456.

Hüne, M. \& C. Ojeda (2012). Estructura del ensamble de peces costeros de los canales y fiordos de la zona central de la Patagonia chilena (48 $-52^{\circ}$ S). Revista de Biología Marina y Oceanografía 47, 451-460.

Jones, J. B. (1998). Distant water sailors: Parasitic Copepoda of the open ocean. Journal of Marine Systems 15, 207-214.

Kabata, Z. (1967). Morphology of Phrixocephalus cincinnatus Wilson, 1908 (Copepoda: Lernaeoceridae). Journal of the Fisheries Research Board of Canada 24, 515-526.

Kabata, Z. (1969). Phrixocephalus cincinnatus Wilson, 1908 (Copepoda: Lernaeoceridae): morphology, metamorphosis and host parasite relationships. Journal of the Fisheries Research Board of Canada 26, 921-934.

Kabata, Z. (1970). Crustacea as enemies of fishes. In: S. F. Snieszko \& H. R. Axelrod (Eds.), Diseases of Fishes, Book 1, pp. 1-171, T. F. H. Publishers, Jersey City, New Jersey.

Kabata, Z. (1981). Copepoda (Crustacea) parasitic on fishes: problems and perspectives. In: W. H. R. Lumsden, R. Muller \& J. R. Baker (Eds.), "Advances in Parasitology, volume 19", Academic Press, London, pp. 1-71.

Kabata, Z. \& C. R., Forrester (1974). Atherestes stomias (Jordan and Gilbert 1880) (Pisces: Pleuronectiformes) and its eye parasite Phrixocephalus cincinnatus Wilson, 1908 (Copepoda: Lernaeoceridae) in Canadian Pacific waters. Journal of the Fisheries Research Board of Canada 31, 1589-1595.

Kabata, Z. \& J. S. Ho (1981). The origin and dispersal of hake (genus Merluccius: Pisces: Teleostei) as indicated by its copepod parasites. Oceanography and Marine Biology 19, 381-404, figs. 1-4.

Kunz, Y. W. (2004). Developmental biology of Teleost fishes. Springer, The Netherlands.

Lloris, D. \& J., Rucabado (1991). Ictiofauna del Canal Beagle (Tierra del Fuego), aspectos ecológicos y análisis biogeográfico. Instituto Español de Oceanografía., Publicaciones Especiales 8, 1-182.

Moreno C. \& F., Jara (1984). Ecological studies on fish fauna associated with Macrocystis pyrifera belts in the south Fueguian Islands, Chile. 
Marine Ecology Progress Series 15, 99-107. Muñoz, G. \& V. Olmos (2007). Revisión bibliográfica de especies ectoparásitas y hospedadoras de sistemas acuáticos de Chile. Revista de Biología Marina y Oceanografía 42, 89-148.

Munz, F. W. (1971). Vision: Visual pigments. En: W. S. Hoar \& D. J. Randall (Eds.), Fish physiology. Academic Press. New York, USA, pp. 1-3.

Navarro, J. \& G. Pequeño (1979). Peces litorales de los archipiélagos de Chiloé y Los Chonos, Chile. Revista de Biologías Marina y Oceanografía 16, 255-309.

Ohtsuka, S., K. K., Harada, T. Miyahara, K. Nagahama, K. Ogawa \& T. Ohta (2007). Prevalence and intensity of Phrixocephalus umbellatus (Copepoda: Siphonostomatoida: Pennellidae) parasitic on Paralichthys olivaceus in the western part of the Sea of Japan. Fisheries Science 73, 214-216.

Pakhomov, E. A., S., Kaehler \& C. D. McQuaid (2002). Zooplankton community structure in the kelp beds of the sub-Antarctic Prince Edward Archipelago: are they a refuge for larval stages? Polar Biology 25, 778-788.

Panella, S., A. Michelato, R. Perdicaro, G. Magazzù, F. Decembrini \& P. Scarazzato (1991). A preliminary contribution to understanding the hydrological characteristics of the Strait of Magellan: Austral Spring 1989. Bollettino di Oceanologia Teorica ed Applicata 2-3, 107-126.

Pequeño, G. (1989). Peces de Chile. Lista Sistemática Revisada y Comentada. Revista de Biología Marina 24, 1-132.

Pequeño, G. (1999). Peces del crucero CIMAR-Fiordo 2, a los canales patagónicos de Chile, con consideraciones ictiogeográficas. Ciencia y Tecnología del Mar 22, 165-179.

Pequeño, G. (2000). Peces del crucero CIMARFiordo 3, a los canales del sur de Magallanes (ca. $\left.55^{\circ} \mathrm{S}\right)$, Chile. Ciencia y Tecnología del Mar 23, 83-94.

Perkins, P. S. (1994). Ultrastructure of the holdfast of Phrixocephalus cincinnatus (Wilson), a blood-feeding parasitic copepod of flatfishes, The Journal of Parasitology 80, 797-804.
Perkins, P. S. \& R. Gartman (1997). Host-parasite relationship of the copepod eye parasite, Phrixocephalus cincinnatus, and Pacific sanddab (Citarichthys sordidus) collected from wastewater outfall areas. Bulletin of the Southern California Academy of Sciences 96, 87-104.

Reyes, P. \& M. Hüne (2012). Peces del Sur de Chile. Ocho Libros Ed. Santiago, Chile. pp. 1-500.

Rohde, K., Ho J. S., Smales L. \& R. Williams (1998). Parasites of Antarctic fishes: Monogenea, Copepoda and Acanthocephala. Marine and Freshwater Research 49, 121-125.

Sandtröm, A. (1999). Visual ecology of fish: a review with special reference to percids. Fiskeriverket Rapport Finland 2, 45-80.

Sielfeld, W. \& M. Vargas (1999). Review of marine fish zoogeography of Chilean Patagonia ( $42^{\circ}$ $57^{\circ}$ S). Scientia Marina 63, 451-463.

Sievers, H, C. Calvete \& N. Silva (2002). Distribución de características físicas, masas de agua y circulación general para algunos canales australes entre el golfo de Penas y el estrecho de Magallanes (Crucero Cimar Fiordo 2), Chile. Ciencia y Tecnología del Mar 25, 17-43.

Silva, N., H. A., Sievers \& R. Prado. (1995). Características oceanográficas y una proposición de circulación, para algunos canales australes de Chile, entre $41^{\circ} 20^{\prime} S$ y $46^{\circ} 40^{\prime}$ S. Revista de Biología Marina 30, 207-254.

Silva N., C. Calvete \& H. A. Sievers (1998). Masas de aguas y circulación general para algunos canales australes entre Puerto Montt y laguna San Rafael (Crucero Cimar Fiordo 1). Ciencia y Tecnología del Mar 21, 17-48.

Valdenegro A. \& N. Silva (2003). Caracterización oceanográfica física y química de la zona de canales y fiordos australes de Chile entre el estrecho de Magallanes y cabo de Hornos (Cimar 3 Fiordos). Ciencia y Tecnología del Mar 26, 19-61.

Wilcock, B. P. \& T. W. Dukes (1989). The eye. In: H.W, Ferguson (Ed.), Systemic Pathology of Fish: A text and atlas of comparative tissue responses in diseases of teleosts, lowa State University Press, Ames, Iowa. 